\title{
Investigation of Mobility Model Against Reactive Routing Protocols in MANETs.
}

\author{
Shruti Bajaj ${ }^{1}$ Er. Rajdeep Singh ${ }^{2}$, Er. Parveen Kakkar ${ }^{3}$ \\ ${ }^{1}$ Department of Computer Science and Engineering PIT -Kapurthala, India \\ ${ }^{2} \mathrm{HOD}$ CSE Department PIT- Kapurthala, India \\ ${ }^{3}$ Assistant Professor DAVIET, Jalandhar, India \\ 1'shrutibajaj826@gmail.com, ${ }^{2}$ ptuap_cse@yahoo.com, ${ }^{3}$ parveen.daviet@gmail.com
}

\begin{abstract}
Ad-hoc network is a collection of wireless mobile nodes which dynamically form a temporary network without the use of any existing network infrastructure or centralized administration. It may connect hundreds to thousands of mobile nodes. The mobile nodes communicate directly with each other without the aid of access points. They form an arbitrary topology, where the routers are free to move randomly and arrange themselves as required. In this paper, an attempt has been made to investigate the impact of mobility models on the performance of three MANET on-demand reactive routing protocols: AODV, DSR and DYMO. The mobility models that are used in this work are: Random Waypoint mobility model and Group mobility model. The performance differentials are analyzed using varying network size, varying pause time, and varying velocity. We used Qual-Net [18] from scalable networks for the simulation purpose. The performance analysis is based on different network metrics such as packet delivery ratio, throughput, average end -to -end delay and average jitter.
\end{abstract}

Keywords: Mobile Ad-hoc Network (MANET) Routing protocol, AODV, DSR, DYMO, Random Waypoint mobility, Group mobility model.

\section{Council for Innovative Research}

Peer Review Research Publishing System

Journal: INTERNATIONAL JOURNAL OF COMPUTERS \& TECHNOLOGY

Vol 8, No 1

editor@cirworld.com

www.cirworld.com, member.cirworld.com 


\section{INTRODUCTION}

A mobile ad-hoc network (MANET) is a self-configuring infrastructure less network of mobile devices connected by wireless links as shown in figure 1. It is a communication network formed from the collection of a number of wireless terminals without the use of any fixed infrastructure. The operation of MANETs does not depend on pre -existing infrastructure or base stations. Therefore each and every node can be treated both as a source as well as destination. The primary challenge in building a MANET is equipping each device to continuously maintain the information required to properly route traffic [1].

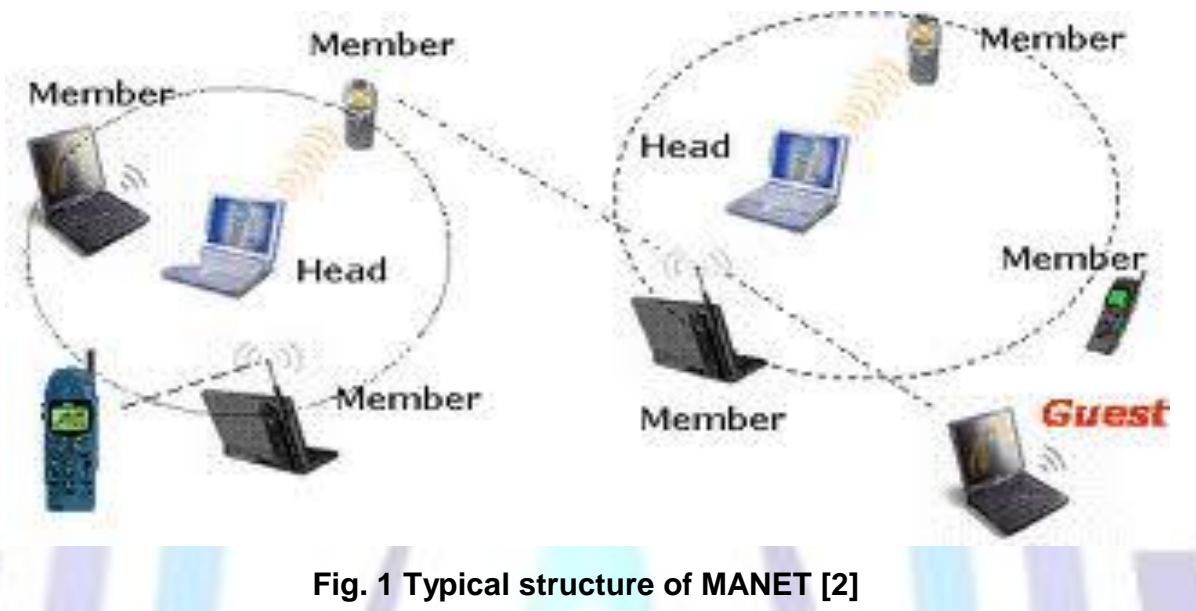

The intent of this paper is to investigate the impact of mobility models on the performance of three reactive routing protocols. Section 1 contains the introduction part. The rest of the paper is organized as follows. Section II discusses routing protocols. Section III describes mobility models. Section IV discusses related work. Section V describes problem statement. Section VI describes present work. Section VII concludes the paper with conclusion and future work.

\section{MANET ROUTING PROTOCOLS}

\section{A. AODV}

AODV is a combination of on-demand and distance vector i.e. hop-to-hop routing methodology [3]. When a node needs to know a route to a specific destination it creates a ROUTE REQUEST. Next the route request is forwarded by intermediate nodes which also create a reverse route for itself for destination. When the request reaches a node with route to destination it creates again a REPLY which contains the number of hops that are require to reach the destination. Al nodes that participate in forwarding this reply to the source node create a forward route to destination. This route created from each node from source to destination is a hop-by-hop state and not the entire route as in source routing.

\section{B. DSR}

DSR is a simple and efficient routing protocol designed specifically for use in multi-hop wireless ad-hoc networks of mobile nodes [3]. It allows nodes to dynamically discover a source route across multiple network hops to any destination in the ad-hoc network. Each data packet sent then carries in its header the complete ordered list of nodes through which the packet must pass, allowing packet routing to be a trivially loop free and avoiding the need for up-to-date routing information in the intermediate nodes through which the packet is forwarded. With the inclusion of this source route in the header of each data packet, other nodes forwarding or overhearing any of the packets may easily cache this routing information for future use.

\section{DYMO}

DYMO is a reactive routing protocol. The basic operations of the DYMO protocol are route discovery and route maintenance. During route discovery Route request (RREQ) message is broadcasted to the network. Every intermediate node participates in hop-by-hop dissemination of this message and records a route to the originator. When a destination node receives this RREQ message, it responds with a Route reply (RREP) message uni-cast towards the originating node. Every node receiving this message creates a route to the destination node and finally this RREP message arrives at the originator of the RREQ message. When a change occurs in the network, topology nodes maintain their routes and monitor their links. When a data packet is received for a route or link that is no longer available the sources of packet generates a Route error (RERR) message and send this RERR message to the packet source to indicate the current route is broken. [15].

\section{MOBILITY MODELS IN MANET}

\section{A. Random Waypoint mobility model}


Random waypoint model was first proposed by Johnson and Maltz is a random mode designed for the movement of mobile users, and how their location, velocity and acceleration change over time [4]. The Random Waypoint Mobility Model includes pause times between changes in direction and/or speed. A mobile node begins by staying in one location for a certain period of time (i.e., a pause time). Once this time expires, the mobile node chooses a random destination in the simulation area and a speed that is uniformly distributed between [min-speed, max-speed].

Figure 2 shows the movement of nodes in a random waypoint mobility model. In this each node moves along a zigzag line from one waypoint $P i$ to the next $P i+1$. The waypoints are uniformly distributed over the given convex area, e.g. unit disk. At the start of each leg a random velocity is drawn from the velocity distribution, (in the basic case the velocity is constant 1). The nodes may have so-called "thinking times" when they reach each waypoint before continuing on the next leg, where durations are independent and identically distributed random variables.

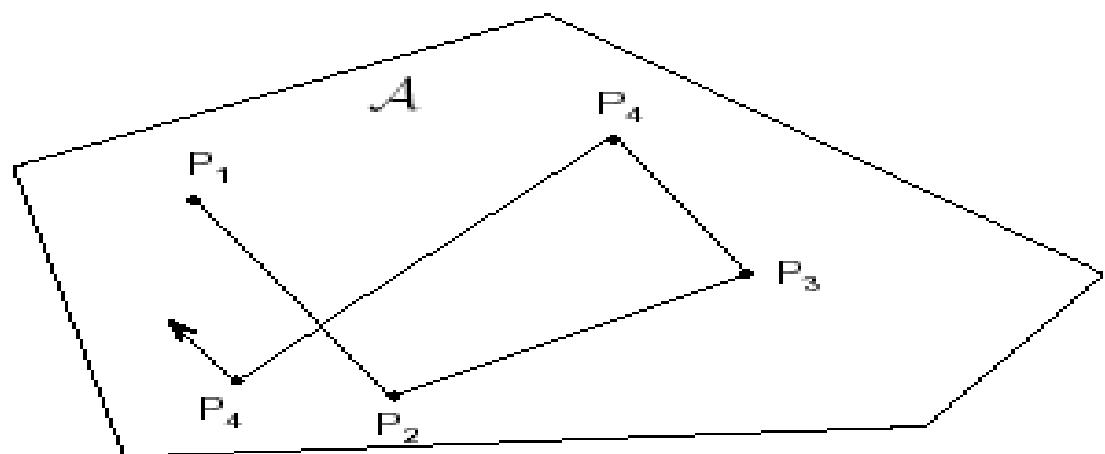

Fig.2 Node movement pattern in Random Waypoint mobility model [5]

\section{B. Group mobility model}

It refers to the scenarios that multiple mobile stations (MSs) move in a together at the same time, generally in the same direction with a short distance of separation. In the normal operation, when the group of MSs moves out of the coverage of the current serving BS and into that of another BS, multiple handovers processes should be performed at almost the same time [10].

In ad-hoc networks, however, there are many situations where it is necessary to model the behavior the mobile nodes as they move together. For e.g., a group of soldiers in a military scenarios may be assigned the task of searching a particular plot of land in order to destroy landmines, capture enemy attackers or simply work together in a collaborative manner to accomplish a common task. For this purpose group mobility models are used. Figure 3 shows the movement of nodes in a group mobility model. In this figure the mobile nodes move in a group. They follow random movement. The nodes move from subgroup BS1 (base station) to subgroup BS 2.

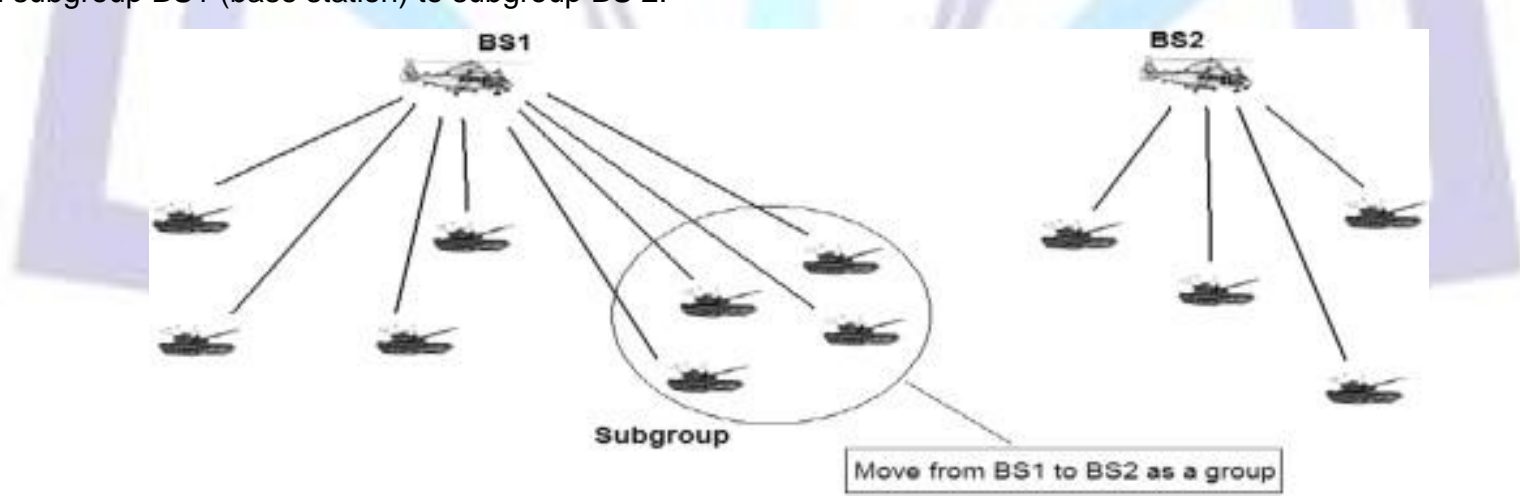

Fig.3 Diagram showing Group mobility model [7]

\section{RELATED WORK}

Fan Bai, et.al, [8] evaluated the impact of different mobility models on the performance of MANET routing protocols. They proposed various protocol independent metrics to capture interesting mobility characteristics, including spatial and temporal dependence and geographic restrictions. A rich set of parameterized mobility models were introduced including Random Waypoint, Group Mobility, Freeway and Manhattan models. They demonstrated the utility of their test suite by evaluating various MANET routing protocols, including DSR, AODV and DSDV. The results showed that the protocol performance may vary drastically across mobility models and performance rankings of protocols may vary with the mobility models used.

Dr.Sridhar Aithal et.al, [9]compared the effect of mobility in case of on-demand reactive routing protocols for mobile ad-hoc network AODV with traditional proactive routing protocol DSDV. The performance was analyzed using varying number of 
connections in the network, mobility pause and speed of the node. The simulations are carried out using NS2 simulator. The results were analyzed for packet delivery ratio, normalized routing and average end-to-end delay by varying the number of connections, speed and pause time.

Shaily Mittal et.al, [10] compared the performance evaluation of three different routing protocols (AODV, DSR and ZRP) in variable pause times of some routing protocols for Mobile Ad-Hoc networks (MANET's). Mobility of the different nodes makes the situation even more complicated. The well known commercial simulator Qual-Net [18] was used to perform simulations. Performance evaluation of AODV, DSR and ZRP is evaluated based on Average end to end delay, TTL based hop count and Packet delivery ratio. Three performance metrics were average end to end delay; average TTL based hop count and packet delivery ratio.

S.R Biradar et.al,[11]compared the performance of two on-demand routing protocols for mobile ad-hoc networks Dynamic source routing (DSR) and Ad-hoc On Demand Vector routing (DYMO). They demonstrated that even though DSR and AODV both are on-demand protocol, the differences in the protocol mechanics can lead to significant performance differentials.

Ashish Shrestha et.al, [12] focused on the performance investigation of reactive and proactive MANET routing protocols, namely AODV, DSR, TORA and OLSR. Hence, the main investigation done in this paper was of the discrete feature and routing in MANET. The simulations were performed using OPNET modeler 14.5. The protocols were tested using the same parameters with high CBR traffic flow and random mobility. Performance of protocols with respect to scalability was also analyzed. The results showed that AODV perform better efficiency to deal with high congestion and scaled better by successfully delivering packets over heavily trafficked network compared to OLSR and TORA.

Sanjay Singh Kushwah et.al, [13] presented investigation regarding the performance comparison of routing protocols for varying node mobility in mobile ad-hoc network (MANETs). The simulated results were observed using NS2. The outcome of the investigation was that reactive protocols perform better than proactive protocols. Further DSR had performed well for the performance parameter namely delivery ration while AODV perform well in terms of average delay. They concluded that both reactive protocols perform well in terms of packet delivery ratio under high mobility scenarios than proactive protocols.

S. Mohapatra et.al, [14] the performance analysis is carried out on Ad-hoc On-demand Distance Vector (AODV), Dynamic Source Routing (DSR), Optimized Link State Routing (OLSR) and Destination Sequenced Distance Vector (DSDV) protocols using NS2 simulator. The delay, throughput, control overhead and packet delivery ratio were the four common measures used for the comparison of the performance of above protocols. The different parameters were number of nodes, different speed of nodes and different size of network. The results concluded that DSR protocol is the best in terms of average PDR. For high mobility condition of nodes DSR gave better packet delivery ratio than other protocols making it suitable for highly mobile random networks.

S. Sagar, et.al, [15] evaluated and compared the performance of two routing protocols, one was reactive, Dynamic MANET on Demand (DYMO) and other was proactive, Optimized Link State Routing (OLSR) in Mobile Ad-hoc Networks (MANETs) and Vehicular Ad-hoc Networks (VANETs). Performance of these protocols was analyzed using three performance metrics; Packet Delivery Ratio, Normalized Routing Overhead and End-to-End Delay against varying scalabilities of nodes. The SUMO simulator was used to generate a random mobility pattern for VANETs. It was observed that DYMO performs better than OLSR for both VANETs and MANETs at the cost of delay. The simulation also resulted that the performance of reactive protocol (DYMODEF and DYMO-MOD) was better than proactive protocol (OLSR-DEF and OLSR-MOD) in both MANETs and in VANETs.

Banoj kumar Panda et.al, [16] described a detailed analysis of performance affected due to change in mobility in different terrain area. The parameter describing the reason of variation in performance was the number of packets delivered. The simulator used in this work for the calculation of performance metric was GLOMOSIM. The performance comparison was made considering two types of routing protocols AODV and DSR. From the analysis it was observed that in the Low terrain area and high density network the number of link break in AODV \& DSR were comparatively less because nodes are confined within a small terrain area and they are within the transmission range of each other. Hence the Packet delivery fraction was less than $100 \%$ because of hidden and exposed terminal problem of MANET, in all mobility condition.

Veena Anand et.al, [17] reported results of NS2 simulation of three important routing protocols: AODV, DSR and DSDV They analyzed performance using average throughput and average end -to-end delay when number of nodes, and also their mobility, was varied. For node movement, a popular model, random waypoint was considered while Constant Bit Rate (CBR) traffic pattern is assumed. Also framework was proposed to analyze the impact of mobility pattern on routing performance of different protocols in MANET through various simulation experiments. They observed that the mobility pattern does influence the performance of MANET routing protocols. The Average Throughput of AODV was found to approach to $91 \%$, the Average throughput of DSDV descends obviously when the routing change was frequent and the routing discovery of DSDV became more difficulty as no. of nodes increases. Hence, AODV and DSR give better Average throughput than DSDV.

\section{PROBLEM STATEMENT}

The objective of this work is to investigate three reactive routing protocols AODV, DSR and DYMO using two mobility models Random Waypoint mobility model and Group mobility model for wireless ad-hoc. The objectives of this paper are outlined as follows: 
- To investigate the impact of mobility on different reactive routing protocols.

- To create different simulation scenarios with varying number of nodes, velocity, and pause times.

- To conduct the performance analysis of random waypoint mobility model and group mobility model on three routing protocols AODV, DSR, DYMO by evaluating parameters viz., throughput, end-to-end delay, packet delivery ratio and jitter.

- To investigate the performance of routing protocols by varying number of nodes, varying pause time and varying velocity using random waypoint and group mobility models.

\section{PRESENT WORK}

Qual-Net is a comprehensive suite of tools for modeling large wired and wireless networks. It uses simulation to predict the behavior and performance of networks to improve their design, operation and management. Qual-Net is a network simulation tool that simulates wireless and wired packet mode communication networks.

\section{A. Simulation Environment}

As already outlined in this paper, an attempt has been made to investigate the impact of mobility models on three reactive routing protocols named AODV, DSR and DYMO. For this two mobility models are used named Random Waypoint mobility model and Group mobility model.

1).RANDOM WAYPOINT MOBILITY MODEL: Random mobility model models the random the random movement of mobile nodes. The simulations are successfully carried out using Qual-Net simulator. For the entire simulations traffic source used is CBR, item size is 512 bytes, queue length is 50 simulation time is 30 seconds transmission range is $250 \mathrm{~m}$ within an area of $1500 \mathrm{~m} * 1500 \mathrm{~m}$.

TABLE I SIMULATION PARAMETERS

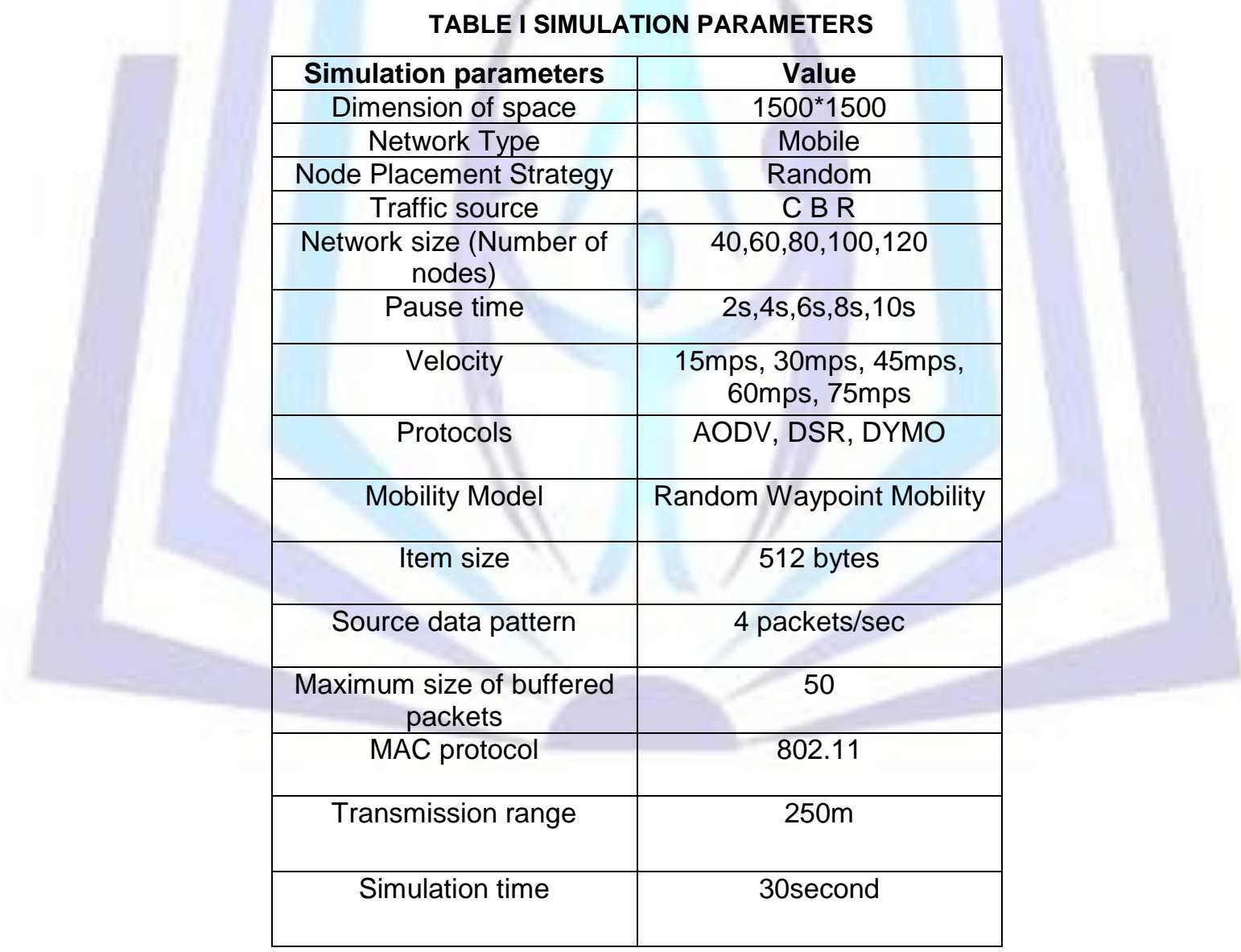

2). GROUP MOBILITY MODEL: Group mobility model makes the movement of mobile nods in a group. Each group has a group leader. For the entire simulations traffic source used is CBR, item size is 512 bytes, queue length is 50 simulation time. In this each group has a logical "center". The center's motion defines the entire group's motion behavior, including location, speed, direction, acceleration, etc. 
TABLE II SIMULATION PARAMETERS

$\left.\begin{array}{|c|c|}\hline \begin{array}{c}\text { Simulation } \\ \text { parameters }\end{array} & \text { Value } \\ \hline \text { Dimension of space } & 1500^{\star} 1500 \\ \hline \text { Network Type } & \text { Mobile } \\ \hline \begin{array}{c}\text { Node Placement } \\ \text { Strategy }\end{array} & \text { C B R } \\ \hline \text { Traffic source } & 40,60,80,100,120 \\ \hline \text { Number of nodes } & 2 \text { s,4s,6s,8s,10s } \\ \hline \text { Pause time } & 15,30,45,60,75 \\ \hline \text { Velocity } & \text { AODV,DSR, DYMO } \\ \hline \text { Protocols } & \text { Group Mobility Model } \\ \hline \text { Mobility Model } & 4 \\ \hline \text { No. of groups } & 512 \text { bytes } \\ \hline \text { Item size } & 4 \text { packets/sec } \\ \hline \text { Source data pattern } & 50 \\ \hline \text { Maximum size of } \\ \text { buffered packets }\end{array}\right)$

The same simulations which were carried out using random waypoint model were also carried out using group mobility model for comparison purposes.

\section{B. PERFORMANCE METRICS}

We investigated and compared the impact of mobility models on routing protocols by evaluating the following four performance metrics:

1). PACKET DELIVERY RATIO: Packet delivery ratio is obtained by dividing the number of packets received by the destination through the number of packets originated by the application layer of the source i.e. (CBR source). It also specifies the packet loss rate, which limits the maximum throughput of the network. The better we obtain the delivery ratio, the more complete and correct is the routing protocol. For an efficient routing protocol it should be high.

2). THROUGHPUT: Throughput may be defined as the amount of data a receiver receives from the sender divided by the time it takes from the receiver to get the last packet. It is usually measured in bits/sec.

C. AVERAGE END-TO-END DELAY: The average End-to-End Delay of a data packet is the time interval when a data packet generated from the CBR source is complete received to the application layer of the destination. For the better performance of a routing protocol it should be low.

D. AVERAGE JITTER: Average Jitter is the time interval between subsequent packet arrivals. This is caused by network congestion, timing drift or route changes. For an efficient routing protocol, the value of jitter should be as low as possible. It is often used as a measure of the variability over time of the packet latency.

\section{RESULTS AND DISCUSSIONS:}

This section discusses the simulation results of relative study of the performance of three reactive routing protocols AODV, DSR and DYMO. This has been done making use of two mobility models Random Waypoint mobility model and Group Mobility model. In order to widely measure the performance of a protocol various network parameters are varied .The simulation in the performance analysis is done by varying number of nodes, pause time and maximum velocity.

\section{A. Random Waypoint Mobility model}

1). VARYING NUMBER OF NODES : The packet delivery ratio graph for MANET routing protocols for varying number of nodes using Random Waypoint Mobility model is shown in figure. 4.1. 


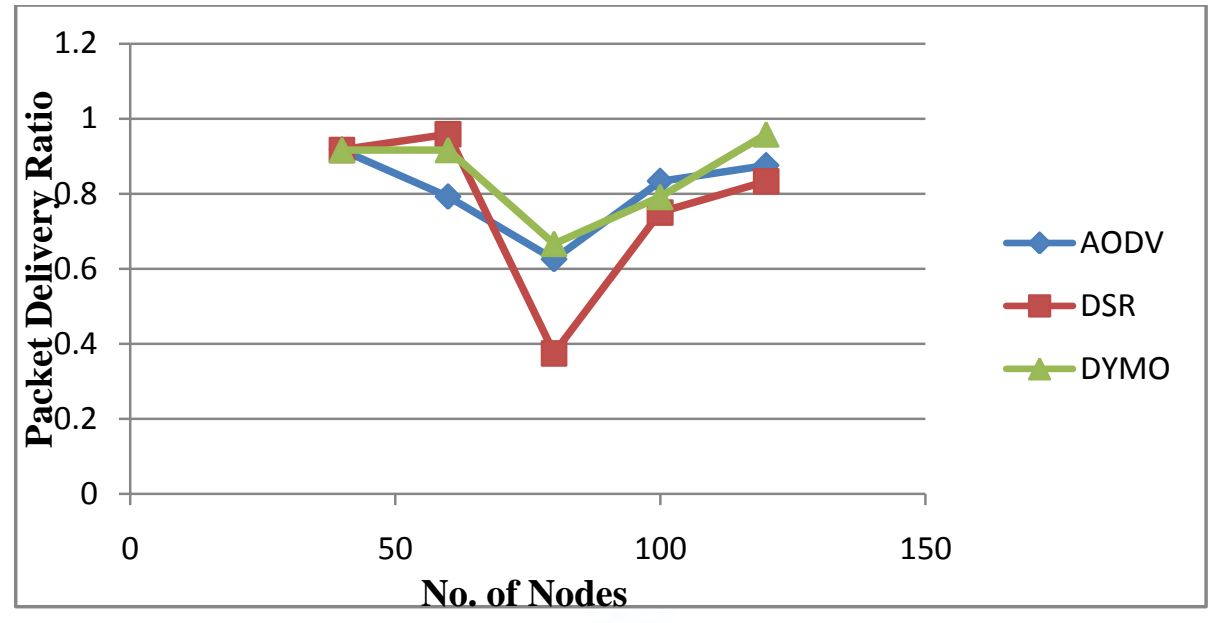

Fig. 4.1 No. of Nodes v/s Packet Delivery Ratio

It is clear that DYMO outperforms both AODV and DSR in terms of delivery ratio. DYMO has the best delivery ratio at every point for nodes. It is also clear that the delivery of AODV and DYMO are found to be similar for varying nodes. Thus, DYMO shows the best performance in terms of delivery for number of nodes.

The throughput for MANET routing protocols for varying nodes shown in figure 4.2. It is found that DSR has lesser value in comparison to AODV and DYMO. The reason for lesser value is that it declines when the number of nodes is 80 . The DYMO has highest throughput in comparison to the other two protocols. As the number of nodes increases DYMO outperforms both AODV and DSR. Thus DYMO shows the best performance in terms of throughput.

The average End-End Delay is shown in figure 4.3. DSR gives an average result in terms of delay and its variation is also low as compared to other routing protocols DYMO demonstrated the worst performance in case of average end- to- end delay. Thus, AODV posses' maximum end-to-end delay and shows the best performance.

The value of average jitter is shown in figure 4.4. Here, we found that DYMO shows the worst performance having minimum value of jitter. This is due to the high packet collision probability more chances to loss packet between subsequent packet transmissions. DSR shows an average value of jitter as compared to the other two protocols whereas AODV shows the best performance in terms of jitter. Thus, AODV posses' minimum average jitter with maximum value of and shows the best performance.

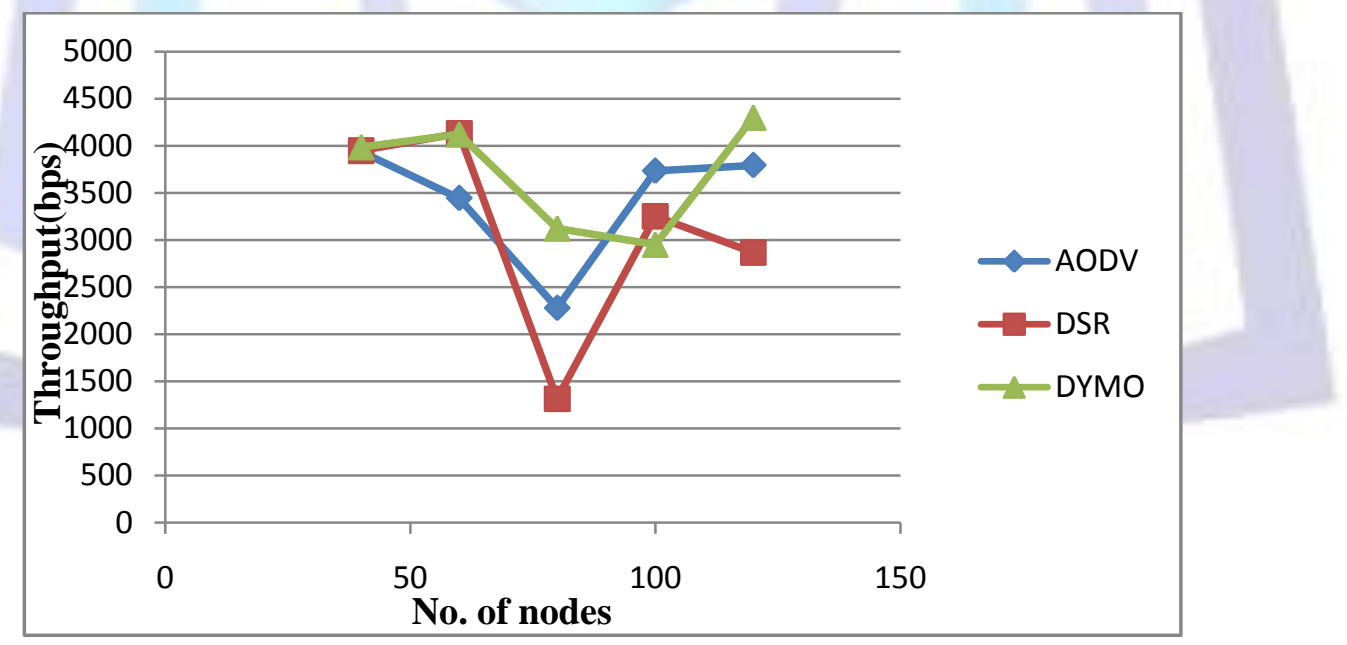

Fig. 4.2 Number of Nodes v/s Throughput 


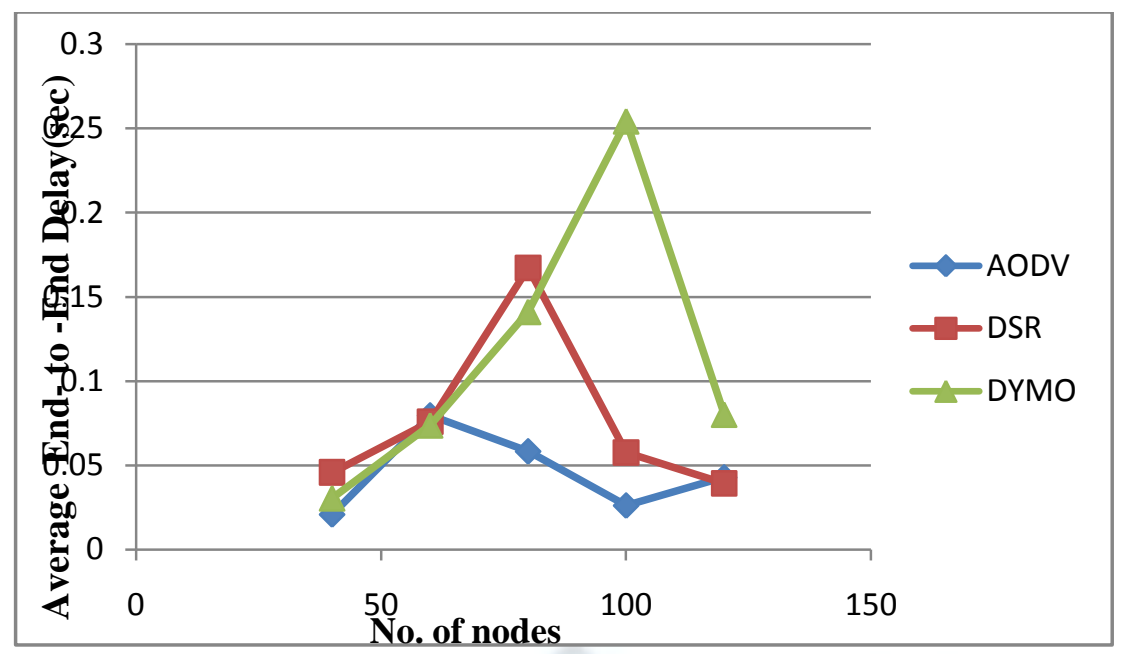

Fig. 4.3 No. of nodes v/s Average Delay

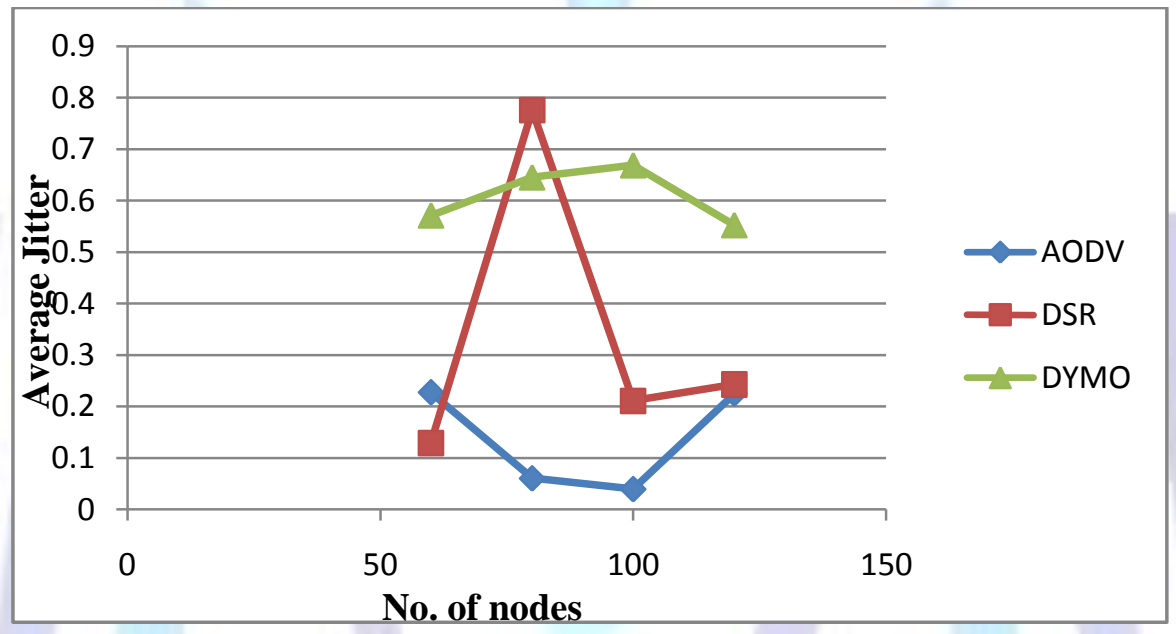

Fig. 4.4 No. of nodes v/s Jitter

2). VARYING PAUSE TIME: The packet delivery ratio graph for MANET routing protocols for variable pause time is shown in figure. 4.5

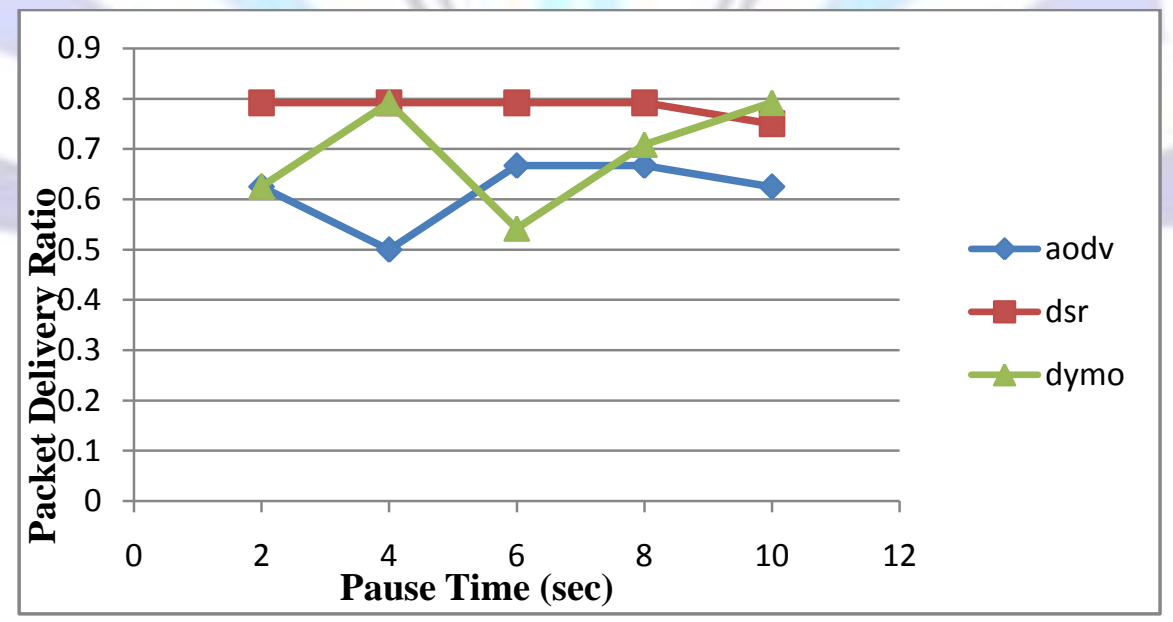

Fig. 4.5 Pause time v/s Packet Delivery Ratio

Here, DSR outperforms both AODV and DYMO i.e. it gives the best delivery ratio at every pause time. It is also clear that the delivery of AODV and DYMO are found to be little similar. Thus, DSR shows the best performance for delivery for the varying pause time. 


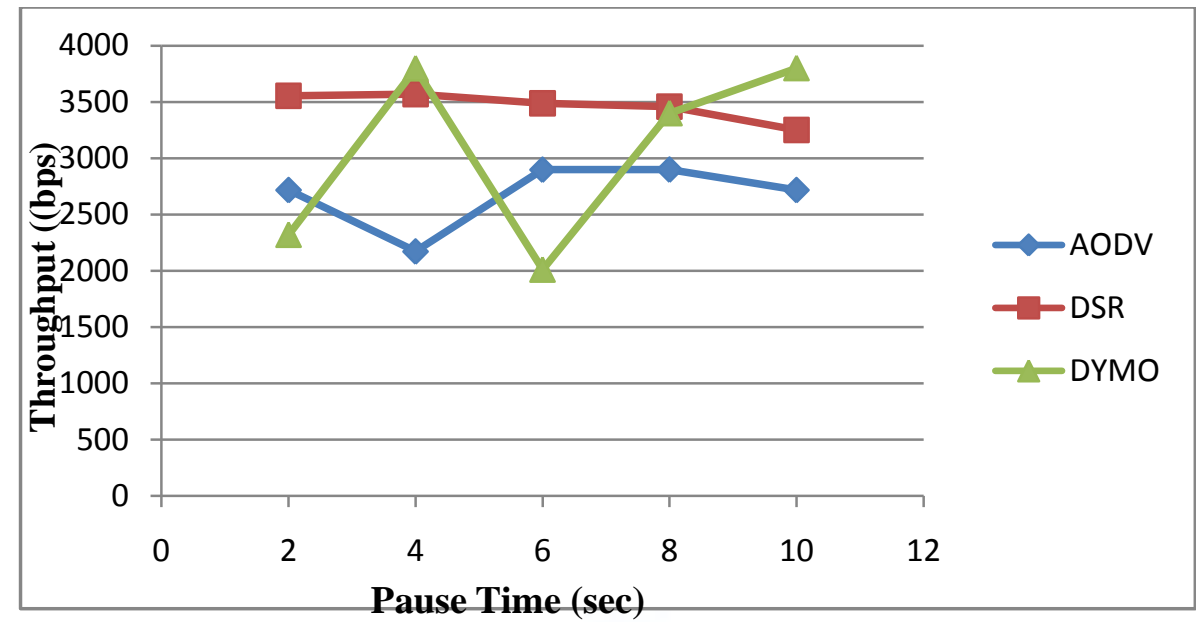

Fig. 4.6 Pause time v/s Throughput

The throughput for MANET routing protocols with respect to pause time is shown in figure 4.6. Our simulation results showed that AODV has lesser value in comparison to DSR and DYMO. The reason is that the value for pause time decreases at 4 seconds which in turn decreases its efficiency. Also, on the increase of pause time, the amount of received packets goes down. DSR has highest throughput. Thus, we conclude that DSR shows the best performance in terms of throughput of for variable pause time. The graph for average end -to-end delay for MANET routing protocols is shown in figure 4.7. From the graph, it is clear that AODV has minimum en-to-end delay with a maximum value. It is also observed that DSR shows an average value of delay whereas DYMO shows the worst performance in case of delay having minimum value of average delay. Thus, AODV performs the best in terms of delay for maximum velocity.

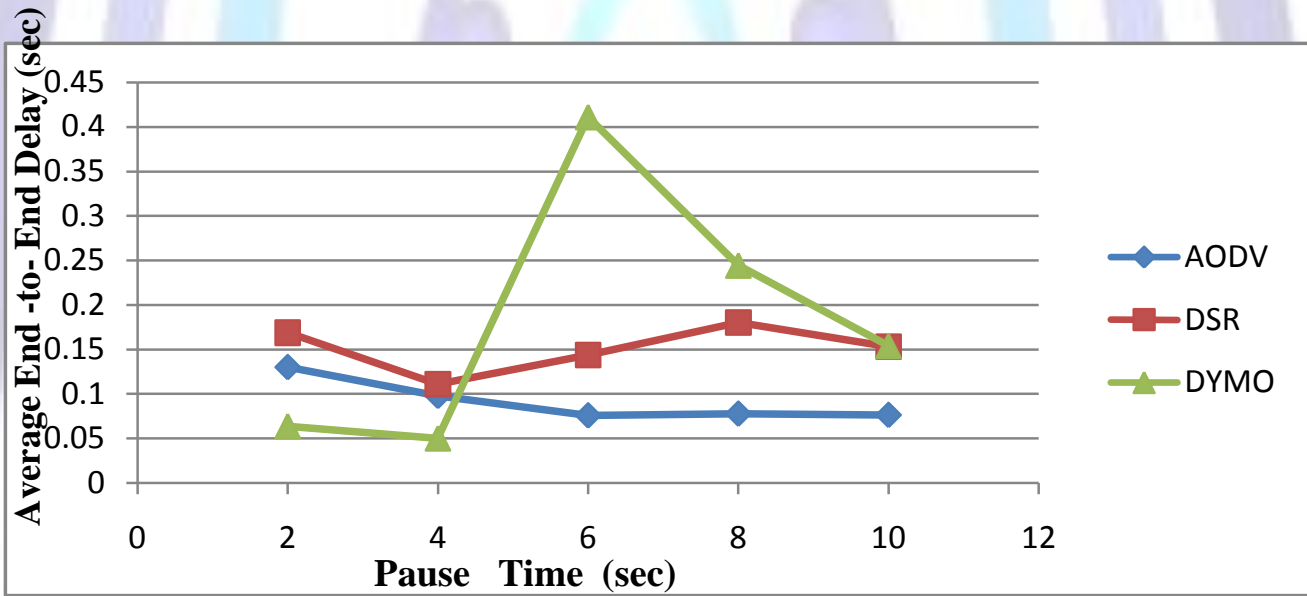

Fig. 4.7 Pause time v/s Average End-to-End Delay

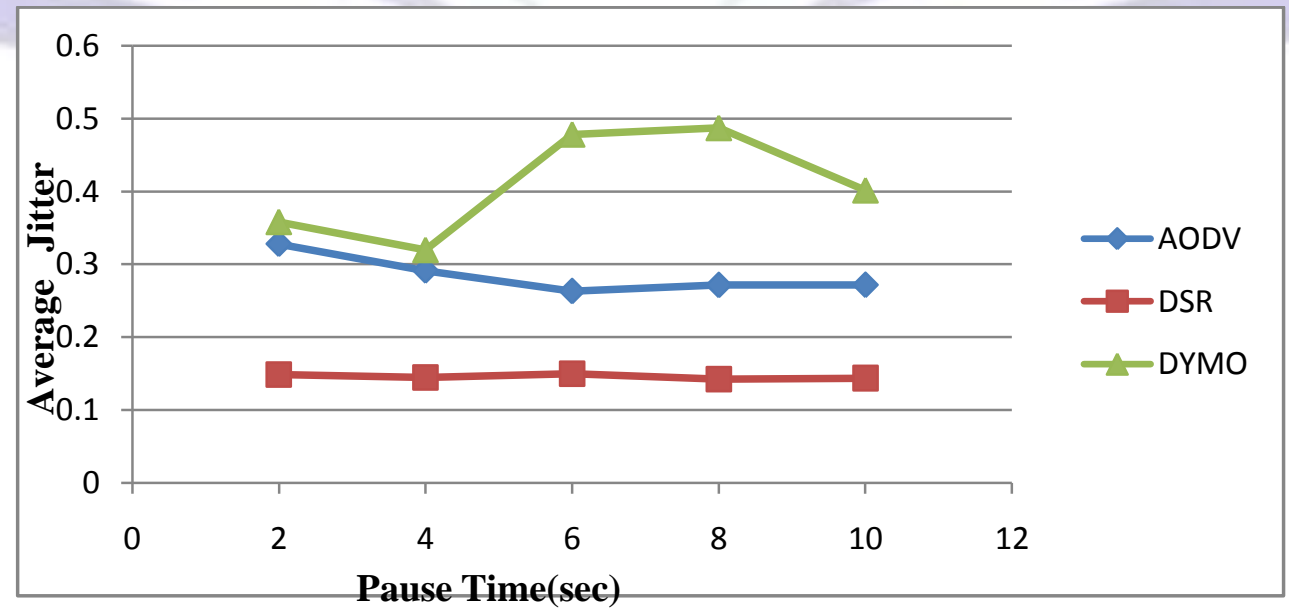

Fig. 4.8 Pause time v/s Average Jitter

The graph for average jitter is shown in figure 4.8. Here, we found that DYMO shows the worst performance having minimum value of jitter. AODV shows an average value of jitter as compared to the other two protocols having value of. 
Hence, it is clear that DSR possess minimum average jitters with maximum value of jitter and thus shows the best performance using random mobility.

3). VARYING VELOCITY: The packet delivery ratio graph for MANET routing protocols for varying velocity using random waypoint mobility is shown in figure 4.9. In this AODV outperforms both DSR and DYMO. It posses maximum delivery ratio for varying velocity. Thus, we finally conclude that AODV gives best performance for delivery ratio for velocity. The graph for throughput with respect to maximum velocity is shown in figure 4.10. DSR shows inferior and lowest throughput as compared to the other two protocols and its throughput is found to remain constant so there is no increase in its performance. There is a great decline in the throughput of DSR when the velocity is $30 \mathrm{mps}$, but at the same time the throughput of AODV, DYMO is almost equal. Thus, DYMO shows the best performance for throughput with variable velocity.

The graph for average end -to -end delay for MANET routing protocols is shown in figure.4.11. From the graph it is clear that AODV has minimum end-to-end delay with a maximum value. It is also observed that DSR shows an average value of delay whereas DYMO shows the worst performance having minimum value of because its average delay decreases with increase in velocity. Thus, AODV shows best performance for delay for maximum velocity. The graph for average jitter for maximum velocity is shown in figure 4.12. We found that DYMO shows the worst performance having minimum value of jitter when the maximum velocity is $15 \mathrm{mps}$. The performance of DYMO is found to be totally different, but the values of DSR and AODV are found to be almost similar. Thus, it is finally concluded that AODV posses' minimum average jitter with maximum value of and shows the best performance.

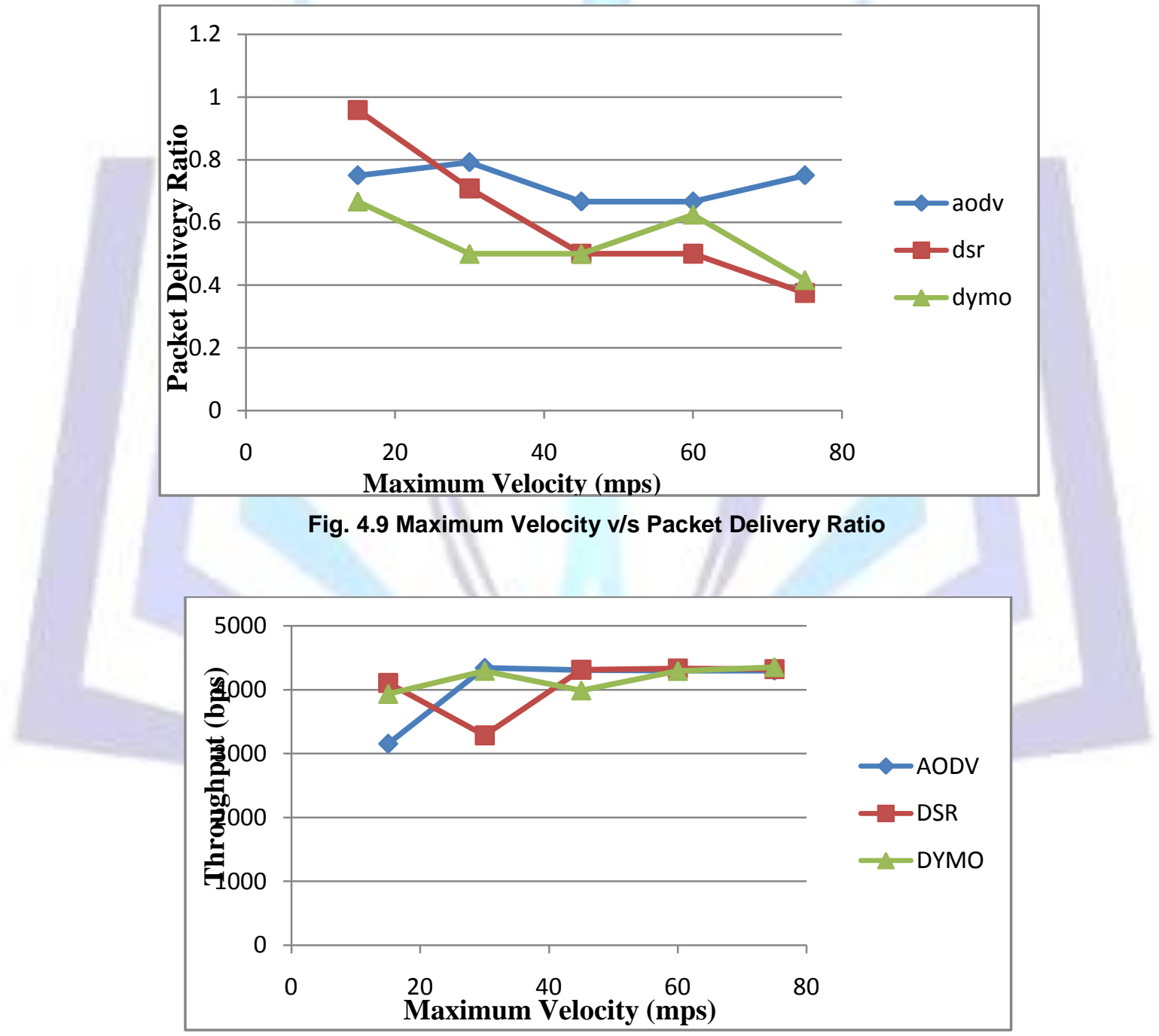

Fig. 4.10 Maximum velocity v/s Throughput 


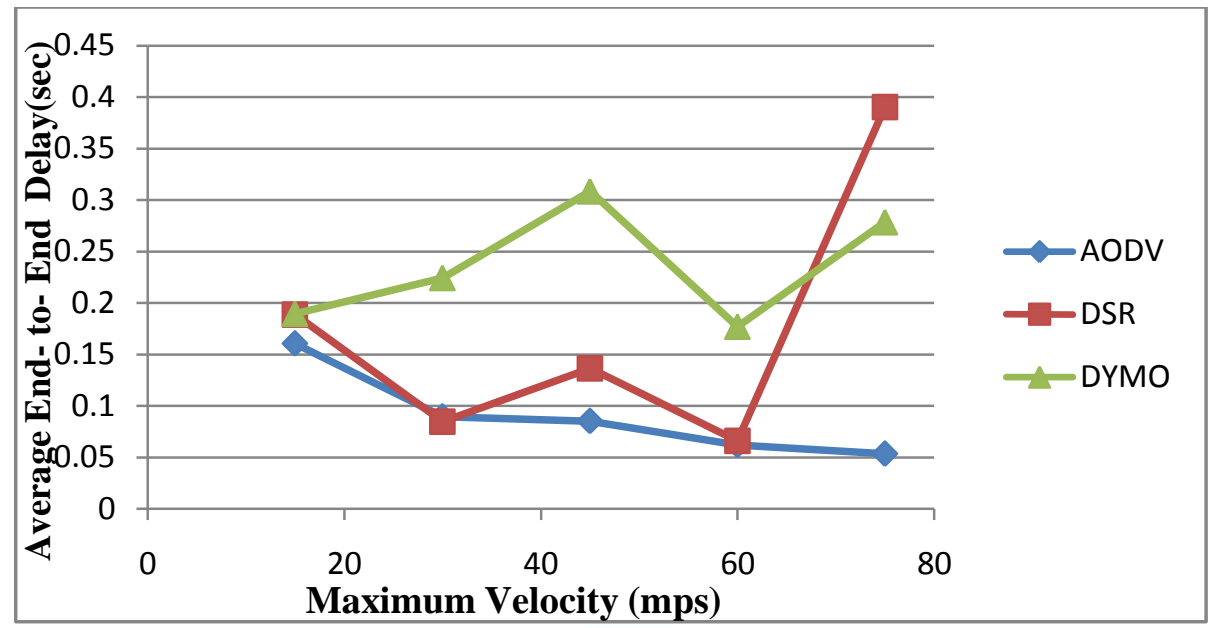

Fig. 4.11 Maximum velocity v/s Average end-to end delay

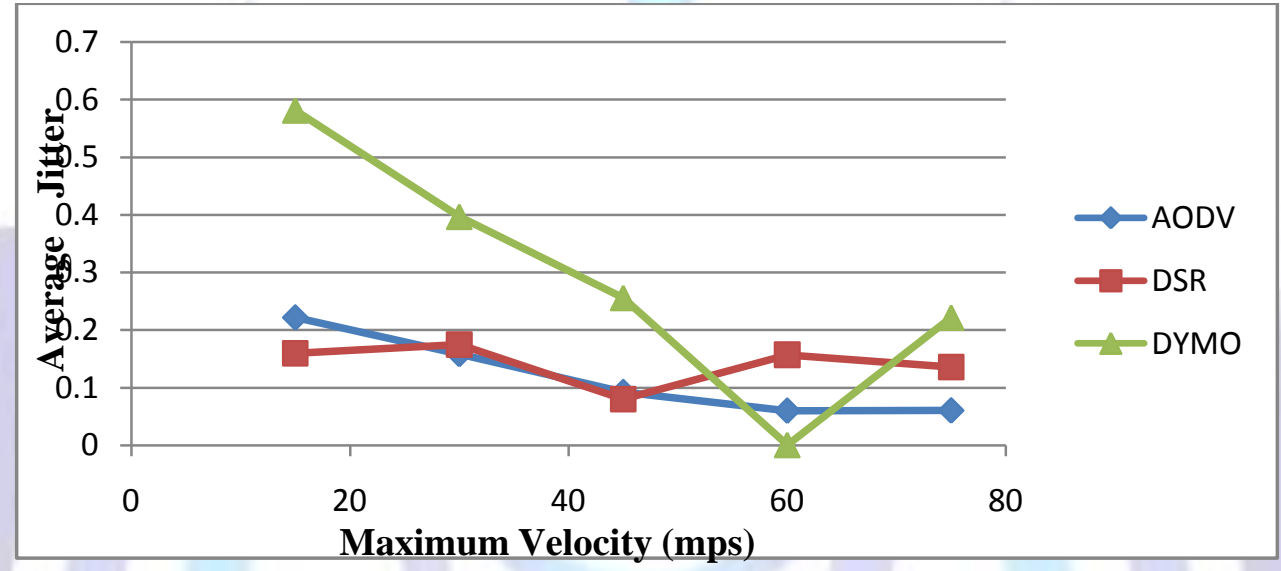

Fig. 4.12 Maximum velocity v/s Average Jitter

\section{B. GROUP MOBILITY MODEL}

1). VARYING NUMBER OF NODES: The packet delivery ratio graph for MANET routing protocols for varying nodes using Group Mobility model is shown in figure 4.13. It is clear that DYMO performs the best for delivery ratio for the varying nodes using Group Mobility model. DYMO posses the maximum delivery ratio at all points of varying number of nodes i.e. with increase in the number of nodes. Thus, DYMO shows the best performance for delivery ratio for nodes using Group mobility.

The throughput for AODV, DSR and DYMO for varying number of nodes using group mobility is shown in figure 4.14. The results show that DYMO outperforms both AODV and DSR. The reason for this is that the value of DYMO increases when the number of nodes increases. With increase in number of nodes the efficiency of both DYMO and AODV protocols also increase. But at the same time the efficiency of DSR is found to decrease. Thus, DYMO shows the best performance in terms of throughput with varying nodes. The average End-End Delay is shown in figure 4.15. In this DSR gives an average result in terms of delay. DYMO demonstrated the worst performance. From nodes 40 to 80 the value of DYMO increases, but the values of DSR and AODV are almost similar. After 80 nodes the delay for DYMO decreases but again the value of AODV and DSR are almost same. Thus, AODV shows best performance for varying nodes with maximum delay. The graph for average jitter is shown in figure 4.16. Here, we found that DYMO show the worst performance having minimum value of jitter. DSR shows an average value of jitter as compared to the other two protocols. Thus, AODV shows the best performance in terms of jitter with maximum value and shows the best performance. 


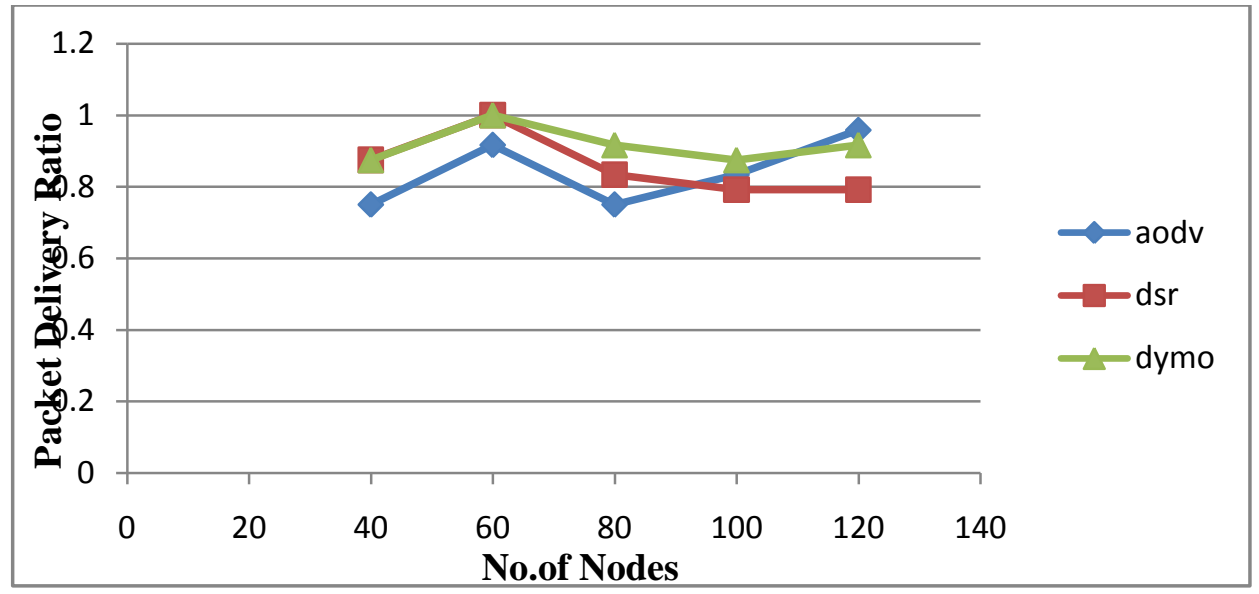

Fig. 4.13 Number of nodes v/s Packet Delivery Ratio

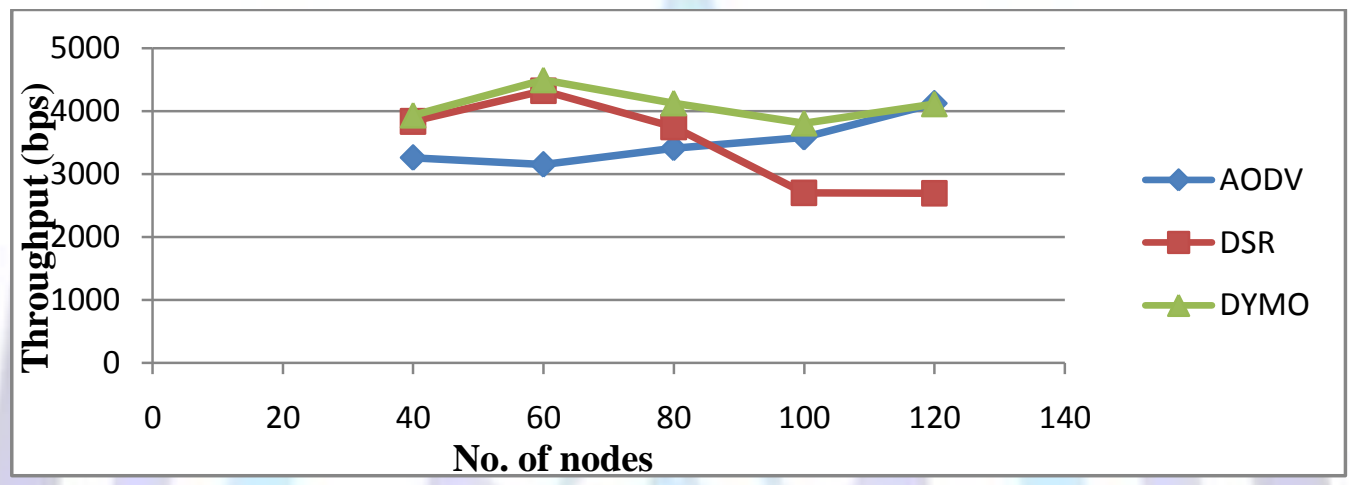

Fig. 4.14 No. of nodes v/s Throughput

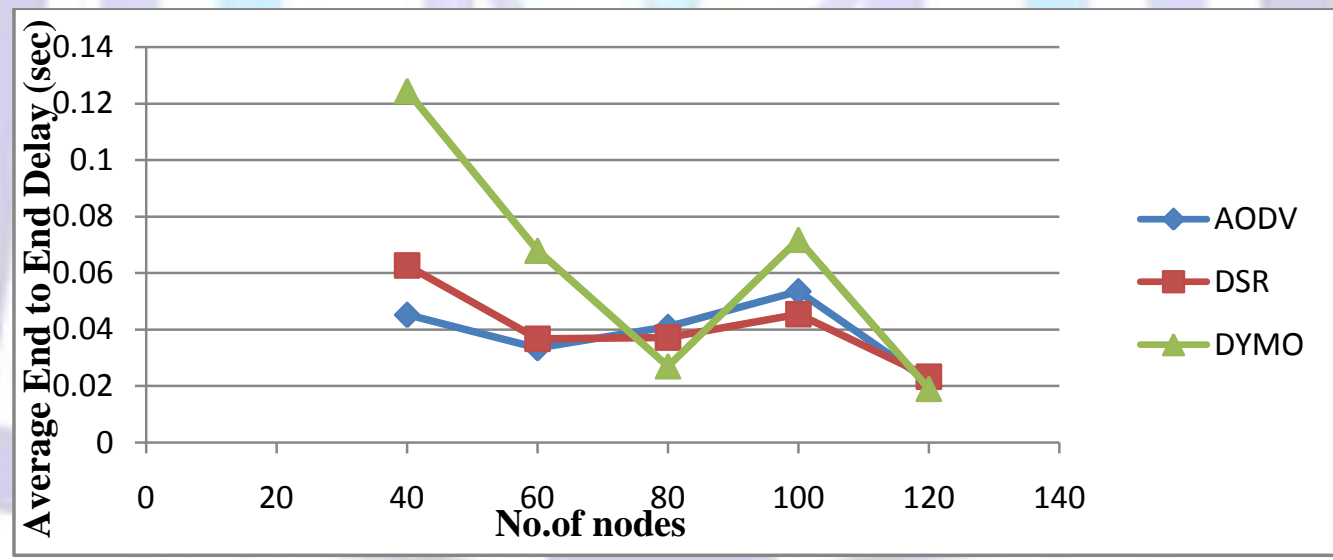

Fig.4.15 No. of nodes v/s Average End-to End Delay

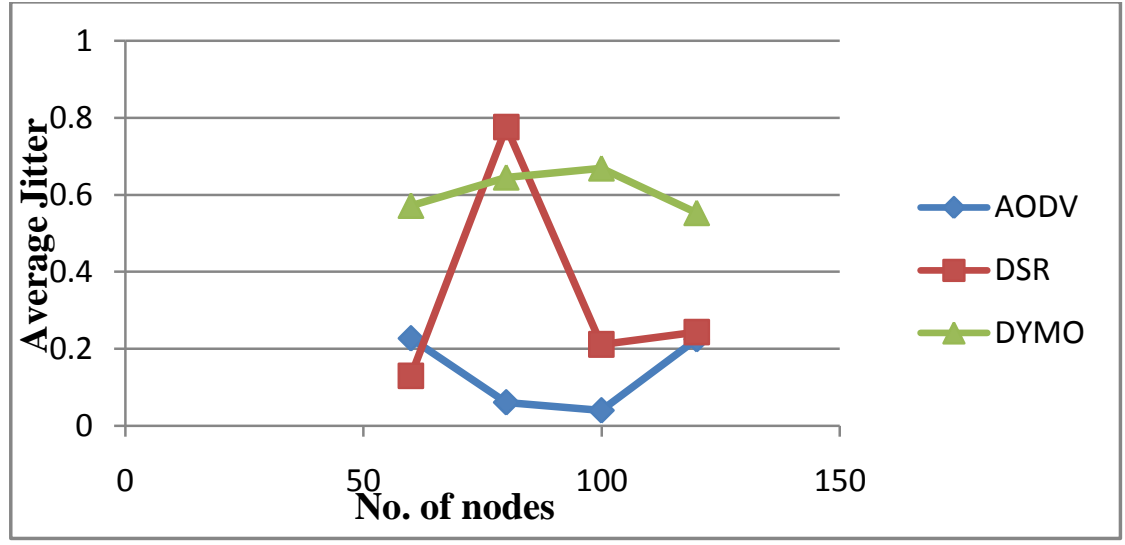

Fig. 4.16 No. of nodes v/s Jitter 


\section{2). VARYING PAUSE TIME:}

The packet delivery ratio graph for MANET routing protocols for varying pause time is shown in figure 4.17 . It is clear that DSR performs the best in terms of delivery ratio for varying pause time using Group Mobility model. It posses the maximum delivery ratio with increase in pause time. The value of DSR remains constant with increase in pause time. Pause time has no effect on the delivery ratio of DYMO with group mobility model. Thus, DSR shows best performance for delivery for varying pause time. The throughput for AODV, DSR and DYMO protocols with respect to pause time is shown in figure 4.18. It is clear that AODV outperforms DSR and DYMO. DYMO protocol is said to have average value of throughput. DSR is said to have lowest value of throughput. Thus, AODV shows the best performance for variable pause time.

The graph for average End-to-End Delay is shown in figure 4.19. Here, it is found that DSR shows an average result in terms of delay. DYMO here also demonstrated the worst performance in case of average end to end delay with value of .In case of DYMO theirs is a drastic change in delay when the pause time increases form 6 seconds to 8 seconds. With the increase in pause time, the average delay will slightly decrease with an incremental increment of pause time. The DYMO and AODV protocol are found to have same value throughout the entire values of pause time. There is no change in the delay of these protocols. But DSR is showing fluctuations at various points. It is the only protocols amongst three which is being affected by pause time. Thus, from the above discussion we can conclude that AODV is found to posses' minimum end-to-end delay with maximum value of and hence shows the best performance. The graph for average jitter for AODV, DSR and DYMO for variable velocity is shown in figure 4.20. In this, we found that DYMO shows the worst performance having minimum value of jitter. AODV shows an average value of jitter as compared to the other two protocols. DSR protocol here also outperformed both the protocols, by showing the best performance in terms of jitter. Thus, DSR possess minimum average jitter with maximum value of, and hence gives the best performance.

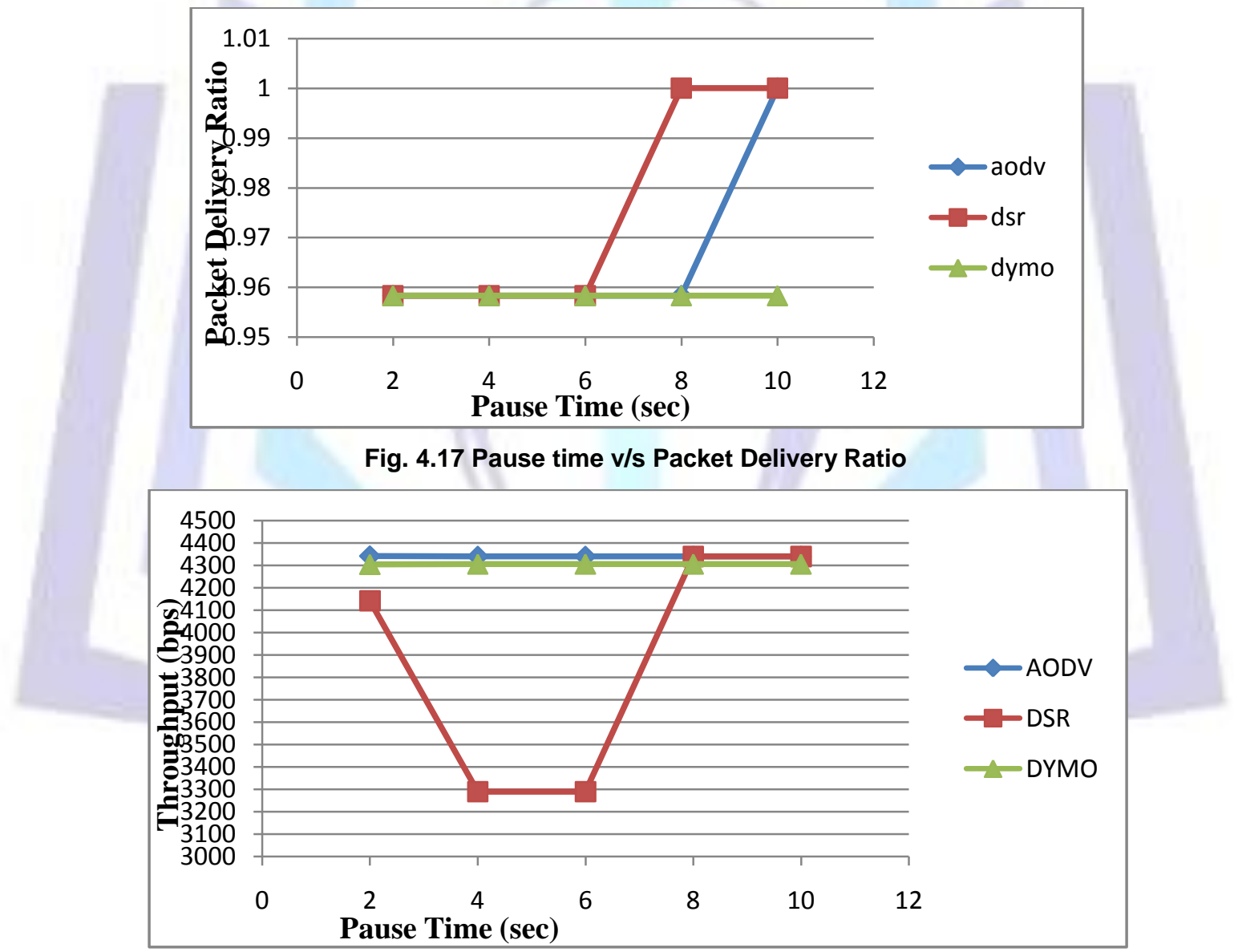

Fig. 4.18 Pause time v/s Throughput 


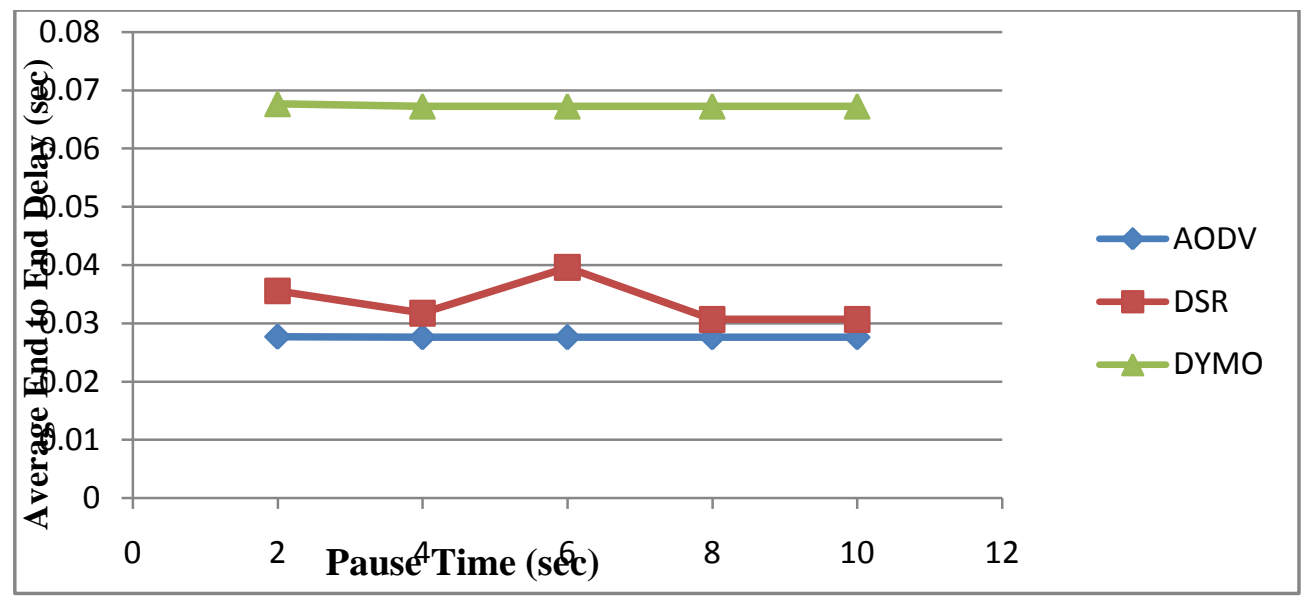

Fig. 4.19 Pause time v/s Average end-to-end delay

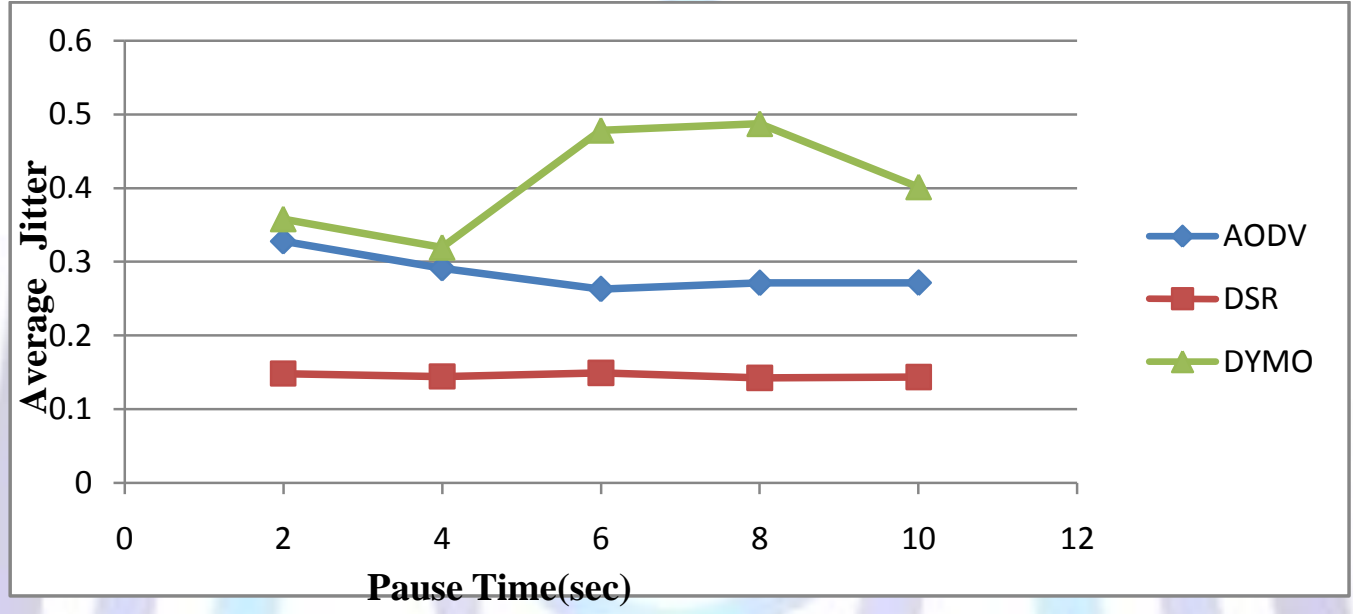

Fig. 4.20 Pause time v/s Average Jitter

3). VARYING VELOCITY: The packet delivery ratio graph for MANET routing protocols for varying velocity using Group Mobility is shown in figure 4.21. It is clear from the graph that AODV protocol performs the best in terms of delivery ratio for variable velocity i.e. with increase in velocity. DSR here gives intermediate performance compared to the other two protocols. DYMO in this gives low value because its value first increases, then decreases, then remains constant and then finally increases. So, overall its performance degrades. Thus, from the above discussion, it can be concluded that AODV shows the best performance in terms of delivery for variable velocity using Group Mobility model.

The graph for throughput with respect to maximum velocity is shown in figure 4.22. In this, DYMO shows inferior and lowest throughput as compared to the other two protocols. As its velocity increases from 40 to 120 , the throughput of DYMO is found to decrease and so its performance declines. We also find that there is a great decline in the throughput of DSR when the velocity increases from 10 to $50 \mathrm{mps}$. DSR here shows an average throughput which is closer to that of DYMO and both have throughput less than AODV .Thus, AODV shows the best performance in terms of throughput with variable maximum velocity.

The graph for average end-to-end delay for maximum velocity is shown in figure 4.23. DSR gives an average result in terms of delay and its variation is also to most extent similar to AODV protocol. DYMO demonstrated the worst performance, and its value is minimum at the initial point when the maximum velocity is 20 mps. The performance of DYMO is found to be totally different, but the values of DSR and AODV are almost similar. Thus in all scenarios, we found that AODV protocol outperforms for average delay.

The graph for average jitter for maximum velocity is shown in figure 4.24. We found that, DYMO performs the worst and has minimum jitter. This is due to the great decline in jitter when the velocity increases from 15 to 60 . The performance of DYMO is found to be totally different, but the values of DSR and AODV are found to be almost similar. DSR shows the best performance in terms of jitter and has value Thus, from the above discussion finally we conclude that AODV possess minimum average jitter with maximum value of and shows the best performance. 


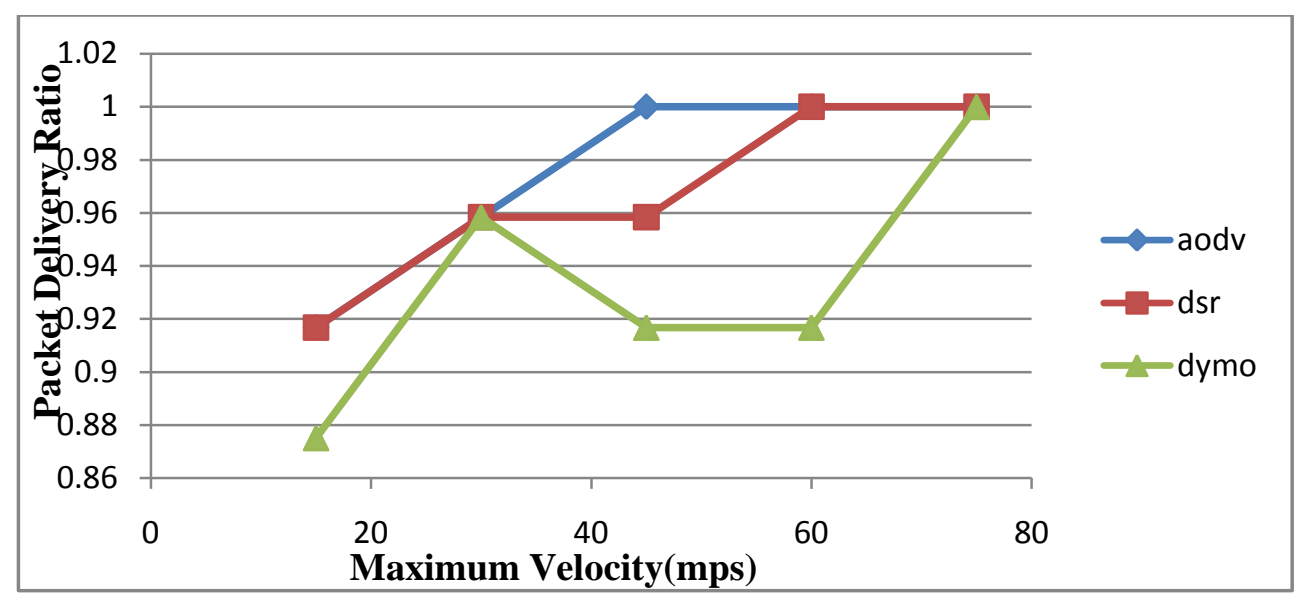

Fig.4.21 Maximum Velocity v/s Packet Delivery Ratio

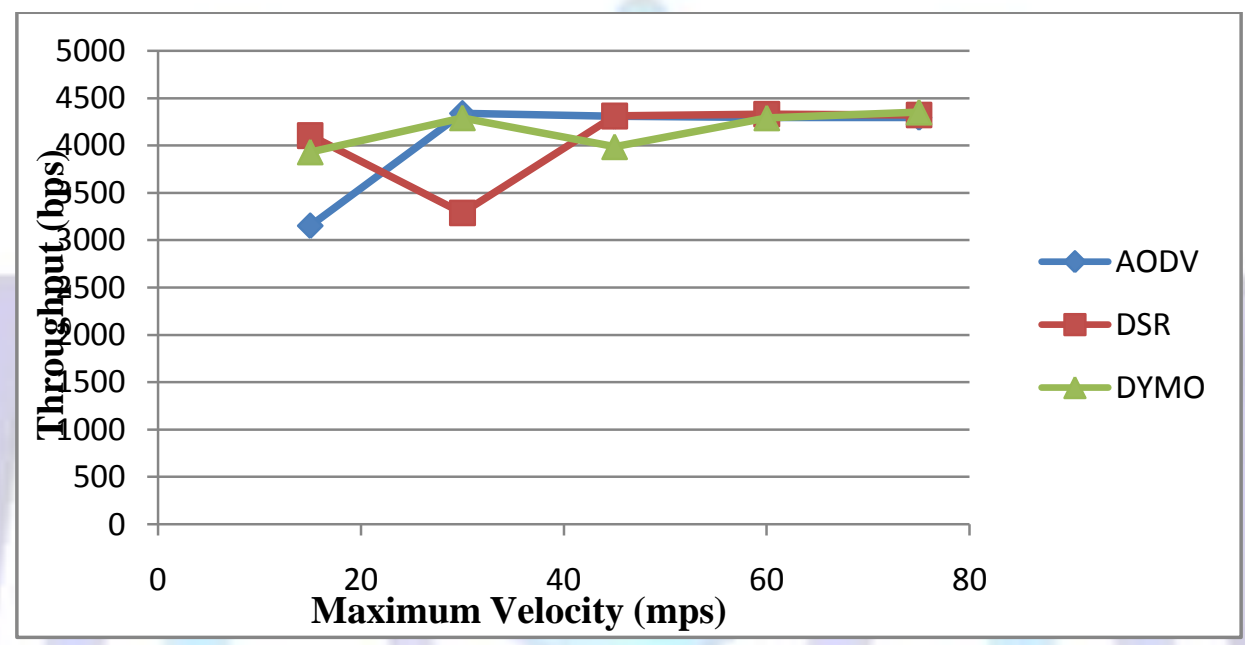

Fig. 4.22 Maximum Velocity v/s Throughput

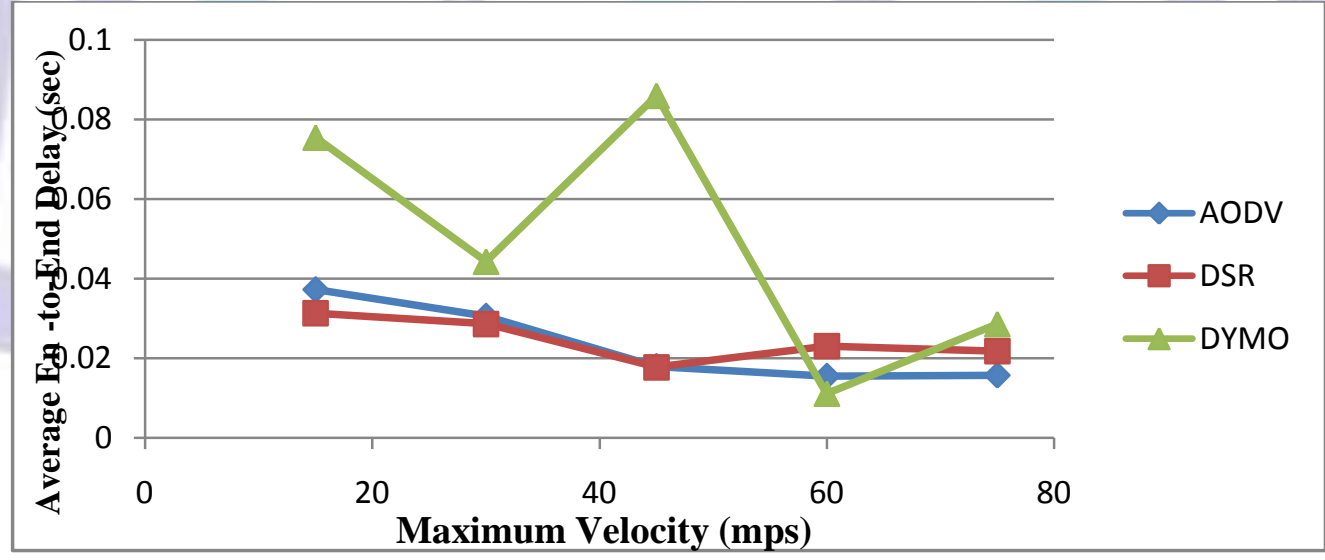

Fig. 4.23 Maximum Velocity v/s Delay 


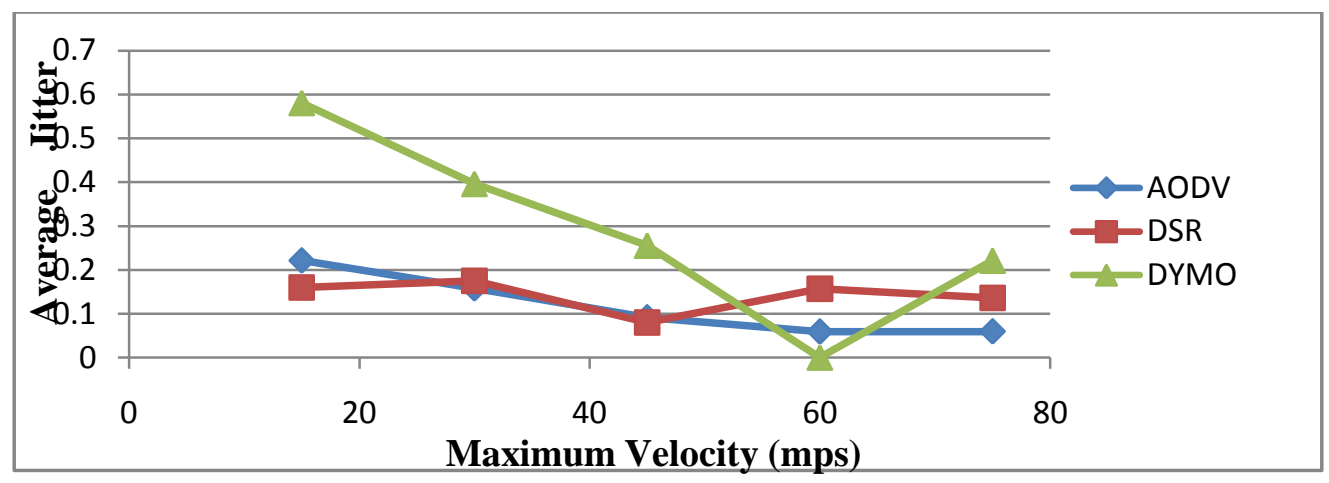

Fig. 4.24 Maximum velocity v/s Average Jitter

\section{CONCLUSION WORK}

This work has compared the performance of AODV, DSR and DYMO using two mobility models Random Waypoint Mobility model, and Group Mobility model using Qual-Net simulator. All the protocols use reactive routing approach. They perform well under high mobility conditions. For the packet delivery ratio, all the protocols perform equally for all the scenarios using both mobility models. The mobility models are not affected by PDR. In case of throughput, for random waypoint mobility model the three protocols perform equally, but under group mobility DYMO better than AODV, and DSR. For the end-to-end delay, only AODV is the best protocol in all scenarios of all the mobility models. For jitter, under random waypoint mobility DSR perform better than other two protocols, but under group mobility AODV perform better than other to protocols. In this work different simulation scenarios are generated with varying nodes, varying pause time and varying velocity. We also observed that pause time does not affect much with group mobility model. In these work network parameters such as simulation area, traffic type-CBR is kept constant.

Future work of MANET routing protocols involves the implementation of new routing protocols. Other protocols can also be taken into account such as TORA, LDR and ZRP, LANMAR, LAR with respect to different metrics including network size, node density and pause time. In our simulation part we did not make simulation of these protocols with respect to energy. Future works can also be geared towards designing MANET environments in another simulator, i.e using Qualnet Cyber Xeta which supports additional features of MANET routing protocol such as energy models, battery models etc. Performance metrics such as normalized routing overhead, Control overhead can also be considered as future work.

\section{REFERENCES}

[1] Homepage on MANETs. [Online].Available: http://en.wikepedia.org/wiki/Mobile_ad_hoc_network.

[2] Homepage on MANETs.[Online].Available: http +architecture=1\&flz=1WIGGNI_en\&tbnm.

[3] S. Mohapatraa, P.Kanungo, "Performance analysis of AODV, DSR, OLSR and DSDV Routing protocols," International Conference on Communication Technology and System Design 2011 Engineering 30 (2012) 69 - 76 in Protocols using NS2 Simulator," in Procedia Engineering 30, 2012, paper 69 - 76 pp 69-76.

[4]Homepage on Mobility.

[Online]. Available: http://en.wikipedia.org/wiki/Mobility_model.

[5][Online].Available:http://www.google.co.in/search?hl=en\&site=imghp\&tbm=isch\&source=hp\&biw

[6][Online].Available:http://citeseerx.ist.psu.edu/viewdoc/summary?doi=10.1.1.138.971

[7][Online].Available:http://www.google.co.in/imgres?q=group+mobility+model\&hl=en\&biw=1366\&bih=588\&tbm=isch\&tbni $\mathrm{d}=\mathrm{y} 1 \mathrm{kuAPfK}$.

[8] Fan Bai, Narayanan Sadagopan, and Ahmed Helmy "A framework to systematically analyze the Impact of Mobility on Performance of Routing protocols for Ad-hoc Networks “,0-7803-7753-2/03/\$17.00 (C) 2003 IEEE INFOCOM 2003

[9] Mrs. Geeta V, Dr. Sridhar Aithal, and Dr. K. ChandraSekaran "Effect of Mobility over Performance of the Ad hoc Networks “, IEEE INFOCOM 2006.

[10] Shaily Mittal and Prabhjot Kaur "Performance Comparison of AODV, DSR and ZRP Routing Protocols in MANET'S ", IEEE INFOCOM 2009.

[11] S. R. Biradar1, Hiren H D Sarma, and Kalpana Sharma ," Performance Comparison of Reactive Routing Protocols of MANETs using Group Mobility Model" International Conference on Signal Processing Systems 978-0-7695-3654-5/09 \$25.00 IEEE 2009.

[12] Ashish Shrestha and Firat Tekiner," International Conference on Parallel and Distributed Computing", Applications and Technologies, International Conference on Signal Processing Systems 978-0-7695-3654-5/09 \$25.00 IEEE 2009. 
[13] Sanjay Singh Kushwah and G.S. Tomar," Investigation of the effects of mobility on routing protocols in MANETs " IEEE International Conference on Ubiquitous Computing and Multimedia Applications, 2011 IEEE, DOI 10.1109/UCMA.2011.26 pp1-3.

[14] S. Mohapatraa, P.Kanungo ," Performance analysis of AODV, DSR, OLSR and DSDV Routing Protocols using NS2 Simulator " International Conference on Communication Technology and System Design Procedia Engineering 30 (2012) pp $69-76$.

[15] S. Sagar, J. Saqib, A. Bibi, N. Javaid ," Evaluating and Comparing the Performance of DYMO and OLSR in MANETs and in VANETs "IEEE INFOCOM, 2011.

[16] Banoj kumar Panda1, Manoranjan Das2, Benudhar Sahu3 ,Rupanita Das," Impact of Mobility and Terrain Size on Performance of AODV and DSR in Mobile Ad-hoc Network" 978-1-4673-1989-8/12/\$31.00 IEEE INFOCOM, 2012.

[17] Performance of AODV, DSR and DSDV Protocols under varying node Movement by Veena Anand and Suresh Chandra Gupta IEEE INFOCOM, 2012.

[18] The Qual-Net simulator www.Scalable.Networks.com.

\section{Author' biography}

\section{Shruti Bajaj:}

She is an M.Tech student in Computer science and Engineering at PIT- Kapurthala. She did her B.Tech also in Computer Science and Engineering from DAVIET, Jalandhar.

\section{Er. Rajdeep Singh}

He received a B.Tech in Computer Science and Engineering from Punjab Technical University, and M.Tech from Punjab Engineering College Chandigarh. He is currently working as a head in the Department of Computer Science and Engineering at PIT (PTU Main Campus). His research includes Mobile Computing and Wireless Sensor Networks.

\section{Er. Parveen Kakkar}

He has done B.Tech in Computer science and Engineering and M.Tech also in Computer Science and Engineering. He is currently working as an Assistant Professor at DAVIET, Jalandhar. His areas of interest include Information Security. 